\title{
HYPOTHYROIDISM AND THE HEART
}

Maja Udovcic, M.D.; Raul Herrera Pena, M.D.; Bhargavi Patham, M.D., Ph.D.; Laila Tabatabai, M.D.; Abhishek Kansara, M.D., M.P.H.

Houston Methodist Hospital, Houston, Texas

\begin{abstract}
Hypothyroidism is a commonly encountered clinical condition with variable prevalence. It has profound effects on cardiac function that can impact cardiac contractility, vascular resistance, blood pressure, and heart rhythm. With this review, we aim to describe the effects of hypothyroidism and subclinical hypothyroidism on the heart. Additionally, we attempt to briefly describe how hypothyroid treatment affects cardiovascular parameters.
\end{abstract}

\section{Introduction}

Hypothyroidism affects between $4 \%$ and $10 \%$ of the population, and the prevalence of subclinical hypothyroidism is reported to be as high as $10 \%$ in various studies..$^{1-3}$ Hypothyroidism is diagnosed when low levels of the thyroid hormones result in elevated levels of thyroid-stimulating hormone (TSH), whereas subclinical hypothyroidism is diagnosed when TSH levels are elevated above the upper limit of the assay reference range with normal thyroid hormone levels. Thyroid hormones play an important role in the normal function of heart and vascular physiology, and hypothyroidism produces profound cardiovascular effects. Of recent clinical interest is the effect of subclinical hypothyroidism on cardiovascular disease and whether or not it should be treated. This review attempts to highlight the effects of hypothyroidism and subclinical hypothyroidism on the heart and current treatment strategies.

\section{Cardiometabolic Changes in Hypothyroidism}

Thyroid hormones have a variety of effects on the cardiovascular system that can greatly impact cardiac function (Figure 1). Hypothyroidism is associated with decreased cardiac output due to impaired relaxation of vascular smooth muscle and decreased availability of endothelial nitric oxide. This produces a cascade effect of increased arterial stiffness that leads to increased systemic vascular resistance. On a molecular level, these alterations result from reduced expression of sarcoplasmic reticulum $\mathrm{Ca}^{2+}$-ATPase and increased expression of phospholamban, which inhibits ATPase. Thyroid hormones also impact

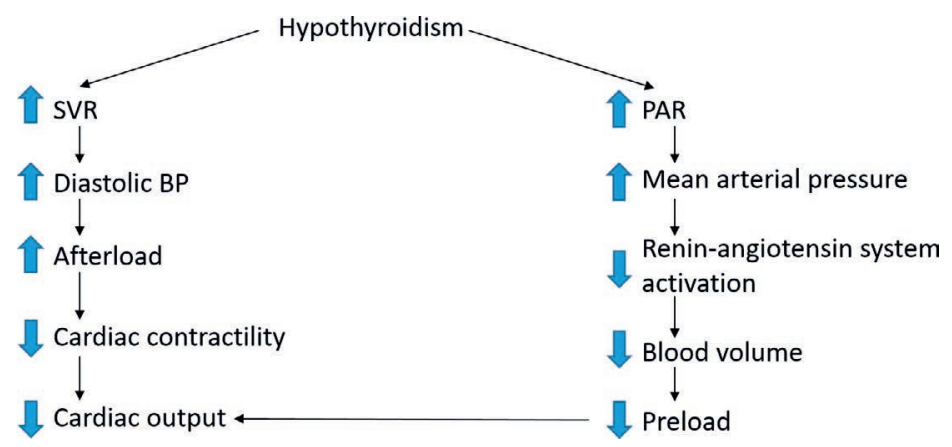

Figure 1. Effects of hypothyroidism on the heart. Modified from Klein et al. ${ }^{1}$ SVR: systemic vascular resistance; BP: blood pressure; PAR: peripheral arterial resistance the renin-angiotensin-aldosterone system. Renin substrates are synthesized in the liver under the stimulus of T3. Thus, in a hypothyroid state, diastolic blood pressure increases, pulse pressure narrows, and renin levels decrease. This results in diastolic hypertension that is often sodium sensitive. ${ }^{1}$ Erythropoietin secretion is increased by $\mathrm{T} 3$, which can explain the normochromic, normocytic anemia often found in hypothyroidism. Thyroid hormones also regulate pacemaker-related genes through transcription as well as the beta-adrenergic system in cardiomyocytes. As a result of these mechanisms, heart rate increases in the presence of thyroid hormones and decreases in hypothyroidism (Table 1). Varied alterations in lipid parameters are noted in both overt and subclinical hypothyroidism, including elevated total cholesterol, low-density lipoprotein (LDL) cholesterol, and apolipoprotein B. A hypothyroid state results in decreased expression of hepatic LDL receptors and reduced activity of cholesterol- $\alpha$-monooxygenase, which breaks down cholesterol, resulting in decreased LDL clearance. ${ }^{4}$ Also noted are elevations in both C-reactive protein and homocysteine (Table 2) ${ }^{1,5}$ Thyroid hormones affect endothelial functions mediated by thyroid hormone receptor (THR)- $\alpha_{1}$ and THR- $\beta$. Activation of THR- $\alpha_{1}$ increases coronary blood flow, decreases coronary resistance in mouse models, and increases production of nitric oxide in endothelial and vascular smooth muscle cells.

\begin{tabular}{|l|l|}
\hline Hemodynamic Effects & Molecular Mechanisms \\
\hline Decreased cardiac output & Endothelial dysfunction \\
\hline $\begin{array}{l}\text { Increased systemic vascular } \\
\text { resistance }\end{array}$ & $\begin{array}{l}\text { Decreased nitric oxide } \\
\text { production }\end{array}$ \\
\hline Decreased arterial compliance & $\begin{array}{l}\text { Decreased vascular smooth } \\
\text { muscle relaxation }\end{array}$ \\
\hline Narrow pulse pressure & Decreased cardiac contractility \\
\hline Increased diastolic pressure & Decreased vascular smooth \\
\hline Atherosclerosis & \\
\hline $\begin{array}{l}\text { Increased risk of coronary } \\
\text { artery disease }\end{array}$ & \\
\hline
\end{tabular}

Table 1. Hemodynamic impact and molecular mechanisms of cardiovascular effects in hypothyroidism. Modified from Klein et al. ${ }^{1}$ 


\begin{tabular}{|l|l|}
\hline $\begin{array}{l}\text { Effect of Hypothyroidism } \\
\text { on Lipids }\end{array}$ & $\begin{array}{l}\text { Molecular Mechanism } \\
\text { of Dyslipidemia in } \\
\text { Hypothyroidism }\end{array}$ \\
\hline Increase in total cholesterol & $\begin{array}{l}\text { Decrease in number of LDL } \\
\text { receptors }\end{array}$ \\
\hline Increase in LDL & $\begin{array}{l}\text { Decrease in LDL receptor } \\
\text { activity }\end{array}$ \\
\hline Increase in apolipoprotein-B & Decrease in LDL clearance \\
\hline Increase in CRP & \\
\hline Increase in homocysteine & \\
\hline
\end{tabular}

Table 2. Lipid alterations and molecular mechanisms in hypothyroidism. Modified from Klein et al. ${ }^{1}$ LDL: low-density lipoprotein; CRP: C-reactive protein

Thyroid hormone activation of THR- $\beta$ induces angiogenesis by initiating the mitogen-activated protein kinase pathway. ${ }_{4}^{4}$ Severe hypothyroidism can also cause pericardial effusion. Though the mechanism is unclear, increased capillary permeability and reduced lymphatic drainage from the pericardial space have been suggested. ${ }^{4}$ Hypothyroidism can also be associated with a decrease in insulin sensitivity due to downregulation of glucose transporters and direct effects on insulin secretion and clearance. $^{6}$

\section{Heart Failure and Hypothyroidism}

As described earlier, hypothyroidism can affect cardiac contractility, which is often diastolic in nature, and impair cardiac muscle relaxation. Associated diastolic hypertension and sometimes-coexistent coronary artery disease further affect myocardial diastolic function. ${ }^{7}$ Cardiac echocardiography has demonstrated impaired relaxation in patients with overt and subclinical hypothyroidism. In addition, early impaired relaxation has been demonstrated by prolongation of the isovolumetric relaxation time and reduction in the $\mathrm{E} / \mathrm{A}$ ratio in subclinical hypothyroidism. ${ }^{8}$ The $\mathrm{E} / \mathrm{A}$ ratio is a ratio of early to late ventricular filling velocities, and a reduced E/A ratio signifies diastolic dysfunction from impaired relaxation. Consequently, it results in a state of low cardiac output with decreased heart rate and stroke volume. It is well known that protein-rich pericardial and/or pleural effusion often occurs in hypothyroidism as a result of increased vascular permeability. In advanced heart failure and shortly after myocardial infarction, the conversion of T4 to T3 decreases. Since T3 is the main regulator of gene expression in myocardial muscle, this decrease has been thought to affect myocardial contractility and remodeling. ${ }^{7}$ Low free T3 levels also have been associated with increased mortality in patients with heart disease. ${ }^{9}$

\section{Arrhythmia and Hypothyroidism}

It is well known that hyperthyroidism is associated with atrial fibrillation (AF). Similarly, hypothyroidism is associated with increased cardiovascular risk factors as well as subclinical and diagnosed cardiovascular disease, both of which are thought to predispose one to AF. However, the relationship between hypothyroidism and AF was evaluated in the Framingham Heart Study and was not found to be statistically significant. ${ }^{10}$ The QT interval is often prolonged in hypothyroidism due to prolonged ventricular action potential. ${ }^{11}$ This is indicative of increased ventricular irritability and in turn can lead to acquired Torsades de pointes. Varying degrees of atrioventricular block and low QRS complexes are also seen in patients with hypothyroidism. ${ }^{12}$ Generally, the incidence of ventricular fibrillation is decreased in hypothyroidism, and depression of thyroid hormone levels appears to be beneficial in patients with angina and acute myocardial infarction. Bradycardia can be beneficial as it raises the arrhythmogenic threshold, especially in patients with underlying cardiovascular disease. In summary, unlike hyperthyroidism, hypothyroidism is linked with a decreased probability of cardiac arrhythmias. ${ }^{12}$

\section{Amiodarone and Hypothyroidism}

Amiodarone is an iodine-rich benzofuranic compound that is used to manage cardiac arrhythmias. ${ }^{13}$ It contains about $37 \%$ iodine by weight, ${ }^{14}$ and a $200-\mathrm{mg}$ dose exposes the patient to about 300 times the recommended daily allowance. ${ }^{15,16}$ Due to its lipophilic nature, it concentrates in several tissues and organs, including the thyroid gland. Its active dealkylated metabolite, desethylamiodarone, tends to have a half-life of about 57 days, thus effects can be long-lasting even after discontinuation. ${ }^{16}$ Amiodarone-induced thyroid dysfunction could be secondary to iodine load or intrinsic effects of amiodarone itself. The iodine load from amiodarone can result in amiodarone-induced hypothyroidism $(\mathrm{AIH})$ by inhibiting the oxidation of iodine, known as the Wolff-Chaikoff effect. ${ }^{17}$ This is particularly the case in patients with underlying or preexisting thyroid disease such as goiter or autoimmune thyroid disease.

Amiodarone by itself can also affect deiodinase activity. In vivo, it inhibits deiodinase- 1 activity and pituitary deiodinase- 2 activity, resulting in high T4, high reverse T3, and low T3. This may spur an initial increase in TSH that often returns to baseline in 2 to 3 months. With long-term treatment, TSH usually normalizes with total and free T4 and reverse T3 levels that remain slightly elevated. Early AIH may be present in $10 \%$ to $20 \%$ of patients treated with amiodarone, whereas the incidence with long-term treatment is often lower, from $5 \%$ to $10 \%$. $\mathrm{AIH}$ is noted to be more frequent in iodine-sufficient areas of the world, ${ }^{18}$ and the presence of thyroid autoantibodies is associated with an increased likelihood of developing AIH. There is also an understanding that amiodarone may hasten the pathogenesis of Hashimoto thyroiditis. Since AIH manifests as primary hypothyroidism, diagnosis is similar to primary hypothyroidism, as is treatment with levothyroxine replacement (Figure 2). ${ }^{16}$

\section{Subclinical Hypothyroidism and the Heart}

Subclinical hypothyroidism is biochemically defined as a TSH level above the upper limit of the reference range with normal thyroid hormone levels. Severity of subclinical hypothyroidism is further defined based on the elevation in TSH levels, where mildly increased serum TSH levels range from $4 \mathrm{mIU} / \mathrm{L}$ to $10 \mathrm{mIU} / \mathrm{L}$, and anything above $10 \mathrm{mIU} / \mathrm{L}$ indicates severely increased TSH levels. ${ }^{6}$ The reported prevalence of subclinical hypothyroidism depends on several factors, such as iodine supplementation, age, and race. In the United States, the prevalence of subclinical hypothyroidism is reported as $4.3 \%$ in the NHANES III study and as high as 9.5\% in the Colorado study. ${ }^{19}$ The annual risk of progression to overt hypothyroidism is reported at $1 \%$ to $5 \%$, depending on TSH levels and thyroid antibody status. Up to $60 \%$ of patients with subclinical hypothyroidism can return to euthyroidism over 5 years, again based on TSH levels and antibody status. ${ }^{19}$ As described earlier, cardiovascular changes of arterial compliance, diastolic blood pressure, endothelial dysfunction, and hyperlipidemia that are noted with overt hypothyroidism can also occur in subclinical hypothyroidism. ${ }^{19}$ 


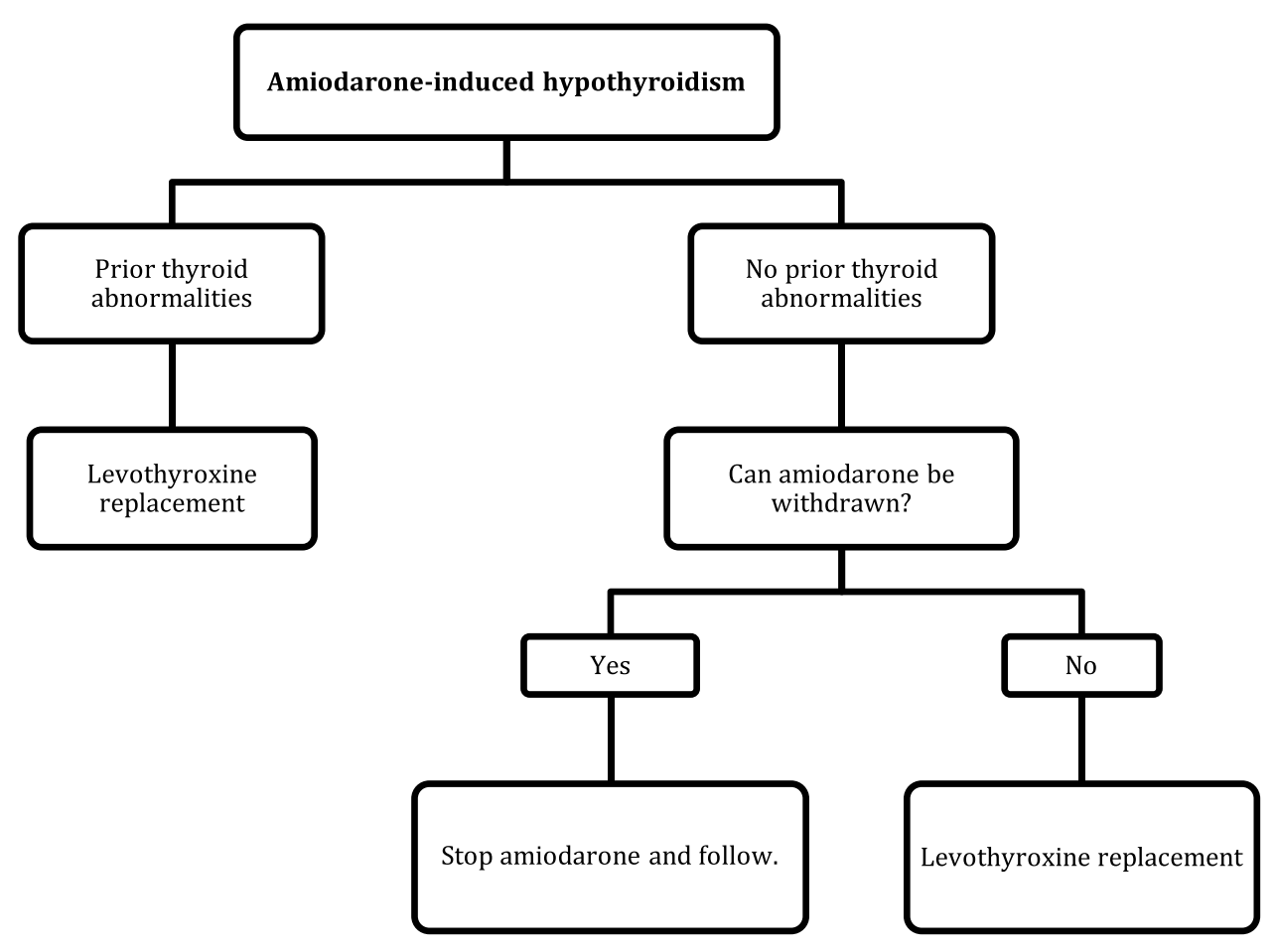

Figure 2. Management of amiodarone-induced hypothyroidism.

Researchers have noted a significant association between subclinical hypothyroidism and prevalence of ischemic heart disease in residents of Nagasaki, Japan, after adjusting for age and sex. The association persisted after additional adjustments were made for other factors such as systolic blood pressure, body mass index, total cholesterol, smoking status, erythrocyte sedimentation rate, and diabetes. ${ }^{20} \mathrm{~A}$ Taiwanese study noted an increased risk for all-cause mortality and cardiovascular death in patients with subclinical hypothyroidism. ${ }^{21}$ One could argue that radiation exposure may have played a role in the Japanese study. However, reanalysis of the United Kingdom-based Whickham survey noted a significantly higher incidence of ischemic heart disease and related mortality in patients with subclinical hypothyroidism as well. ${ }^{22}$ In contrast, the EPIC-Norfolk study did not reveal an increase in coronary heart disease and all-cause mortality across a decade of follow-up. ${ }^{19,23} \mathrm{~A}$ meta-analysis that included 11 prospective cohorts across five continents noted that subclinical hypothyroidism is associated with an increased risk of coronary heart disease events and mortality with elevated TSH levels, especially in those with values greater than $10 \mathrm{mIU} / \mathrm{L} .{ }^{24}$ Several of those studies suggested an increased risk of coronary heart disease and cardiovascular mortality and an increased risk of cardiovascular disease in younger individuals with the cut-off age varying from 50 to 70 years. ${ }^{6}$

\section{Age and Thyroid-Stimulating Hormone}

It is well recognized that the incidence of cardiovascular disease increases with advancing age. At the same time, there is evidence highlighting varying upper limits for a normal TSH reference range. Population studies such as NHANES III and NHANES 1999-2002 found that TSH values are higher with advancing age; however, these studies also indicate that older patients may have higher TSH values in the absence of thyroid disease. ${ }^{25,26}$

\section{Effect of Hypothyroid Treatment on the Heart}

Treatment with levothyroxine in those with overt thyroid dysfunction has been shown to improve LDL cholesterol, total cholesterol, triglycerides, hypertension, diastolic dysfunction, heart rate, and heart rate variability in exercise and to delay progression of atherosclerosis. ${ }^{1}$ Patients with cardiomyopathies may demonstrate improved cardiac contractility and stroke volume with levothyroxine treatment. One of the main concerns with starting levothyroxine replacement is the precipitation of myocardial ischemia or arrhythmias, which, although rare, are known to occur. The recommendation for these patients is usually to start with low doses and gradually escalate until euthyroid status is achieved.

In patients with subclinical hypothyroidism, cohort studies have not found significant differences in cholesterol levels or diastolic blood pressures. However, small randomized trials have discovered some beneficial effects of treatment with levothyroxine. In a study of women with subclinical hypothyroidism, 18 months of treatment resulted in normalization of systolic and diastolic blood pressure and of total and LDL cholesterol as well as decreased carotid intima thickness. ${ }^{27}$ In another study of patients with subclinical hypothyroidism and coronary artery disease, no significant changes occurred in the group randomized to levothyroxine, but those who received placebo had echocardiographic evidence of progression to myocardial diastolic dysfunction. ${ }^{28}$ Although there are no randomized clinical trials evaluating long-term cardiovascular outcomes and mortality in patients treated with levothyroxine, a population-based study of levothyroxine-treated patients demonstrated that those with elevated TSH (defined as greater than $4 \mathrm{mIU} / \mathrm{L}$ ) had a greater risk for cardiovascular events despite receiving the drug. ${ }^{29}$ Analysis from a population-based cohort study revealed that patients with treated hypothyroidism noted no increase in all-cause or cancer mortality but did notice an increase in cardiovascular morbidity in terms of ischemic heart disease and dysrhythmias.

Analysis from the United Kingdom General Practitioner Research Database noted that treatment of subclinical hypothyroidism with levothyroxine was associated with fewer ischemic heart disease events in younger individuals (ages 40-70 years) but not 
notable in older individuals (> 70 years). ${ }^{30}$ Several studies have indicated that levothyroxine treatment in patients with subclinical hypothyroidism has a favorable impact on surrogate markers of vascular disease; however, they do not provide enough confidence that levothyroxine therapy can reverse vascular risk. ${ }^{6}$ The 2013 European Thyroid Association guidelines recommend replacement therapy with levothyroxine in patients younger than 65 years of age with serum TSH levels $>10 \mathrm{mIU} / \mathrm{L}$. The guidelines discourage hormone treatment in the oldest patients (> 80-85 years) with TSH values $<10 \mathrm{mIU} / \mathrm{L}$; however, these patients should be carefully monitored. ${ }^{6}$ These guidelines further recommend that if treatment of subclinical hypothyroidism is undertaken in patients with cardiac disease and in the elderly, a small dose of levothyroxine (25 or $50 \mathrm{mcg}$ ) should be started with gradual titration until a full replacement dose is achieved.

\section{Conclusion}

It is clear that thyroid hormones play a significant role in regulating cardiac, vascular, and metabolic physiology. Physiologic alterations from both overt and subclinical hypothyroidism have varied cardiovascular effects, and treatment may reverse some, if not all, of the effects. There is evidence to suggest that treatment of mild dysfunction can improve cardiovascular outcomes; however, randomized controlled clinical trials in this field are lacking and warranted. It is important to note that TSH levels can be higher in older populations but may not necessitate treatment, and treatment initiated in the elderly should be gradually escalated with close monitoring.

\section{Key Points:}

- Hypothyroidism can result in decreased cardiac output, increased systemic vascular resistance, decreased arterial compliance, and atherosclerosis.

- Impaired cardiac muscle relaxation, decreased heart rate, and decreased stroke volume contribute to heart failure in hypothyroidism.

- Subclinical hypothyroidism is associated with ischemic heart disease and increased cardiovascular mortality.

- Treatment of hypothyroidism may have a beneficial impact on several parameters of cardiac dysfunction, including subclinical hypothyroidism, especially in younger individuals.

Conflict of Interest Disclosure: The authors have completed and submitted the Methodist DeBakey Cardiovascular Journal Conflict of Interest Statement and none were reported.

Keywords: hypothyroidism, subclinical hypothyroidism, thyroxine, thyroid-stimulating hormone

\section{References}

1. Klein I, Danzi S. Thyroid disease and the heart. Circulation. 2007 Oct 9;116(15):1725-35.

2. Ochs N, Auer R, Bauer DC, et al. Meta-analysis: subclinical thyroid dysfunction and the risk for coronary heart disease and mortality. Ann Intern Med. 2008 Jun 3;148(11):832-45.

3. Razvi S, Shakoor A, Vanderpump M, Weaver JU, Pearce SH. The influence of age on the relationship between subclinical hypothyroidism and ischemic heart disease: a metaanalysis. J Clin Endocrinol Metab. 2008 Aug;93(8):2998-3007.

4. Jabbar A, Pingitore A, Pearcs SH, Zaman A, lervasi G, Razvi S. Thyroid hormones and cardiovascular disease. Nat Rev Cardiol. 2017 Jan; 14(1):39-55.
5. Vargas-Uricoechea H, Bonelo-Perdomo A, Sierra-Torres CH. Effects of thyroid hormones on the heart. Clin Investig Arterioscler. 2014 Nov-Dec;26(6):296-309.

6. Pearce SH, Brabant G, Duntas L, et al. 2013 ETA Guideline: Management of Subclinical Hypothyroidism. Eur Thyroid J. 2013 Dec;2(4):215-28.

7. Kahaly GJ, Dillmann WH. Thyroid hormone action in the heart. Endocr Rev. 2005 Aug;26(5):704-28.

8. Rodondi N, Bauer DC, Cappola AR, et al. Subclinical thyroid dysfunction, cardiac function, and the risk of heart failure. The Cardiovascular Health study. J Am Coll Cardiol. 2008 Sep 30;52(14):1152-9.

9. Iervasi G, Pingitore A, Landi P, et al. Low-T3 syndrome: a strong prognostic predictor of death in patients with heart disease. Circulation. 2003 Feb 11;107(5):708-13.

10. Kim EJ, Lyass A, Wang N, et al. Relation of hypothyroidism and incident atrial fibrillation (from the Framingham Heart Study). Am Heart J. 2014 Jan;167(1):123-6.

11. Klein I, Ojamaa K. Thyroid hormone and the cardiovascular system. N Engl J Med. 2001 Feb 15;344(7):501-9.

12. Tribulova N, Knezl V, Shainberg A, Seki S, Soukup T. Thyroid hormones and cardiac arrhythmias. Vascul Pharmacol. 2010 MarApr;52(3-4):102-12.

13. Bogazzi F, Tomisti L, Bartalena L, Aghini-Lombardi F, Martino E. Amiodarone and the thyroid: a 2012 update. J Endocrinol Invest. 2012 Mar;35(3):340-8.

14. Bogazzi F, Bartalena L, Gasperi M, Braverman LE, Martino E. The various effects of amiodarone on thyroid function. Thyroid. 2001 May;11(5):511-9.

15. Batcher EL, Tang XC, Singh BN, Singh SN, Reda DJ, Hershman JM; SAFE-T Investigators. Thyroid function abnormalities during amiodarone therapy for persistent atrial fibrillation. Am J Med. 2007 Oct;120(10):880-5.

16. Cohen-Lehman J, Dahl P, Danzi S, Klein I. Effects of amiodarone therapy on thyroid function. Nat Rev Endocrinol. 2010 Jan;6(1):34-41.

17. Markou K, Georgopoulos N, Kyriazopoulou V, Vagenakis AG. lodine-Induced hypothyroidism. Thyroid. 2001 May;11(5):501-10.

18. Leung AM, Braverman LE. lodine-induced thyroid dysfunction. Curr Opin Endocrinol Diabetes Obes. 2012 Oct;19(5):414-9.

19. Cooper DS, Biondi B. Subclinical thyroid disease. Lancet. 2012 Mar 24;379(9821):1142-54.

20. Imaizumi M, Akahoshi M, Ichimaru S, et al. Risk for ischemic heart disease and all-cause mortality in subclinical hypothyroidism. J Clin Endocrinol Metab. 2004 Jul;89(7):3365-70.

21. Tseng FY, Lin WY, Lin CC, et al. Subclinical hypothyroidism is associated with increased risk for all-cause and cardiovascular mortality in adults. J Am Coll Cardiol. 2012 Aug 21;60(8):730-7.

22. Razvi S, Weaver JU, Vanderpump MP, Pearce SH. The incidence of ischemic heart disease and mortality in people with subclinical hypothyroidism: reanalysis of the Whickham Survey cohort. J Clin Endocrinol Metab. 2010 Apr;95(4):1734-40.

23. Boekholdt SM, Titan SM, Wiersinga WM, et al. Initial thyroid status and cardiovascular risk factors: the EPIC-Norfolk prospective population study. Clin Endocrinol (Oxf). 2010 Mar;72(3):404-10.

24. Rodondi N, den Elzen WP, Bauer DC, et al. Subclinical hypothyroidism and the risk of coronary heart disease and mortality. JAMA. Sep 22;304(12):1365-74.

25. Surks MI, Hollowell JG. Age-specific distribution of serum thyrotropin and antithyroid antibodies in the US population: implications for the prevalence of subclinical hypothyroidism. J Clin Endocrinol Metab. 2007 Dec;92(12):4575-82. 
26. Surks MI, Boucai L. Age- and race-based serum thyrotropin reference limits. J Clin Endocrinol Metab. 2010 Feb;95(2):496-502.

27. Adrees M, Gibney J, El-Saeity N, Boran G. Effects of 18 months of L-T4 replacement in women with subclinical hypothyroidism. Clin Endocrinol (Oxf). 2009 Aug;71(2):298-303.

28. Fadeyev W, Sytch J, Kalashnikov V, Rojtman A, Syrkin A, Melnichenko $G$. Levothyroxine replacement therapy in patients with subclinical hypothyroidism and coronary artery disease. Endocr Pract. 2006 Jan-Feb;12(1):5-17.
29. Flynn RW, Bonellie SR, Jung RT, MacDonald TM, Morris AD, Leese GP. Serum thyroid-stimulating hormone concentration and morbidity from cardiovascular disease and fractures in patients on long-term thyroxine therapy. J Clin Endocrinol Metab. 2010 Jan;95(1):186-93.

30. Razvi S, Weaver J, Butler T, Pearce SH. Levothyroxine treatment of subclinical hypothyroidism, fatal and nonfatal cardiovascular events, and mortality. Arch Intern Med. 2012 May 28;172(10):811-7. 